\title{
On the optical and electrical properties of rf and a.c. plasma polymerized aniline thin films
}

\author{
U S SAJEEV, C JOSEPH MATHAI ${ }^{\dagger}$, S SARAVANAN", RAJEEV R ASHOKAN, \\ $S$ VENKATACHALAM ${ }^{\ddagger}$ and $M$ R ANANTHARAMAN* \\ Department of Physics, Cochin University of Science and Technology, Cochin 682 022, India \\ ${ }^{\dagger}$ Department of Physics, Indian Institute of Technology Bombay, Mumbai 400 076, India \\ ${ }^{\#}$ School of Physics, Environmental and Mathematical Sciences, University of New South Wales at Australian \\ Defence Academy, Canberra, ACT 2600, Australia \\ ${ }^{\ddagger}$ PCM Division, Vikram Sarabhai Space Centre, Thiruvananthapuram 695 022, India
}

MS received 18 July 2005; revised 10 January 2006

\begin{abstract}
Polyaniline is a widely studied conducting polymer and is a useful material in its bulk and thin film form for many applications, because of its excellent optical and electrical properties. Pristine and iodine doped polyaniline thin films were prepared by a.c. and rf plasma polymerization techniques separately for the comparison of their optical and electrical properties. Doping of iodine was effected in situ. The structural properties of these films were evaluated by FTIR spectroscopy and the optical band gap was estimated from UV-vis-NIR measurements. Comparative studies on the structural, optical and electrical properties of a.c. and rf polymerization are presented here. It has been found that the optical band gap of the polyaniline thin films prepared by $\mathrm{rf}$ and a.c. plasma polymerization techniques differ considerably and the band gap is further reduced by in situ doping of iodine. The electrical conductivity measurements on these films show a higher value of electrical conductivity in the case of rf plasma polymerized thin films when compared to the a.c. plasma polymerized films. Also, it is found that the iodine doping enhanced conductivity of the polymer thin films considerably. The results are compared and correlated and have been explained with respect to the different structures adopted under these two preparation techniques.
\end{abstract}

Keywords. Conducting polymers; plasma polymerization; optical band gap; polyaniline; a.c. and rf plasma polymerization; doped polyaniline.

\section{Introduction}

Plasma polymerization or glow discharge polymerization includes a.c., rf and d.c. techniques. It is an excellent and inexpensive tool for growing uniform, ultrathin, amorphous, and pinhole free polymer layers on different substrates. These films are insoluble in organic solvents and resistant to high temperatures. These properties arise from the highly cross-linked structure of polymer thin films evolved under plasma polymerization (Yasuda 1985). They find potential application in various devices (Li and Meichsner 2001; Paterno et al 2002).

Among the various conducting polymers synthesized, polyaniline occupies a prime position, because of its unique characteristics like inexpensiveness of the monomer, ease of processing and excellent stability. It is widely investigated in both thin film and bulk forms, because its electronic and photonic properties are interesting. Polyaniline in its pure and doped forms find extensive applications in

*Author for correspondence (mraiyer@yahoo.com) making devices like polymer light emitting diodes, photovoltaics, sensors, batteries and super capacitors (Joseph Mathai et al 2002). The low dielectric thin films based on a.c. plasma polymerized polyaniline may find applications in the microelectronics industry in the form of inter layer dielectrics (Joseph Mathai et al 2002; Saravanan et al 2004).

It has been reported by various researchers that doping of polyaniline enhances the electrical conducting properties. Dopants like $\mathrm{HCl}$, iodine and CSA, can be incorporated in the polyaniline backbone to produce novel materials for different applications (Skotheim 1986; MacDiarmid 2001). However, reports on the modification of electrical and optical properties of plasma polymerized polyaniline by the incorporation of dopants is less abundant in the literature. It is in this context that a systematic study on the preparation of pure and doped polyaniline thin films by employing both rf and a.c. plasma polymerization techniques is undertaken. The optical and electrical properties of these films are compared and correlated. An attempt is made to explain the observed changes in the conducting and optical properties with respect to the structure adopted during polymerization. 


\section{Experimental}

\subsection{RF plasma polymerization}

Plasma polymerized thin films of aniline on ultrasonically cleaned glass substrates were obtained by polymerizing aniline monomer under radio frequency (rf) plasma discharge in a home built set up. The experimental set up is shown in figure 1a. The rf plasma polymerization unit consists of a deposition cell made up of borosilicate glass tube of about $0.5 \mathrm{~m}$ in length and a diameter of about $0.05 \mathrm{~m}$. The monomer is injected into the chamber by means of a needle valve. Power from a radio frequency oscillator is capacitatively coupled to the deposition chamber by means of aluminium foils wrapped around the glass chamber. The thickness of the films was measured by a Dektak 6M Stylus Profiler. The conditions of polymerization have been standardized and optimized and it has been found that good quality films can be deposited under a monomer vapour pressure of 0.1 Torr and a current of $80 \mathrm{~mA}$ at a frequency of $13.56 \mathrm{MHz}$.

\subsection{A.C. plasma polymerization}

The experimental set up for a.c. plasma polymerization unit (also home built) is depicted in figure 1b. It consists of two parallel stainless steel electrodes, each with diameters of $0.23 \mathrm{~m}$ and placed $0.05 \mathrm{~m}$ apart. Ultrasonically cleaned glass substrates were placed on the lower electrode for the polymer thin film deposition. The a.c. plasma polymerization chamber was evacuated using a rotary pump. Monomer aniline was injected into the glass chamber between the electrodes by means of a glass sprayer at a monomer vapour pressure of 0.2 Torr. Plasma discharge was obtained in the chamber by applying a potential of $500-800 \mathrm{~V}$ at a frequency of $50 \mathrm{~Hz}$, between the electrodes with electrode current lying in the range 50-70 mA. In the present study metal-polymer-metal sandwich structures of cross sectional area, $0.25 \times 10^{-5} \mathrm{~m}^{2}$, were used for the conductivity studies.

\subsection{Doping of thin film samples}

Iodine doping of plasma polymerized aniline thin films was effected by introducing iodine vapour into the plasma polymerization chamber along with the monomer vapour by means of a separate feed through. The introduction of iodine vapour into the chamber was in such a way that this did not affect the pressure inside the vacuum chamber. The thin films prepared in the iodine atmosphere are heated at $330 \mathrm{~K}$ for expelling the unbound iodine on the surface of the film.

\section{$2.4 U V$-vis-NIR and FTIR spectroscopy}

The FTIR spectra of pure and doped rf and a.c. polyaniline thin film samples were recorded by a Nicolet Avatar 360 FTIR Spectrophotometer in the wavelength range $400-4000 \mathrm{~cm}^{-1}$ under identical conditions. The UV-vis-NIR absorption spectra of the samples were recorded using a JASCO-V-570 UV-vis-NIR spectrophotometer. The optical band gap of these samples was evaluated from the photon energy absorption plot.

\subsection{Conductivity measurements}

The room temperature conductivity measurements were carried out using an automated Keithley 236 Source Measurement Unit. For that the Al-polymer-Al structures were mounted inside a home made conductivity cell under dynamic vacuum. (a)

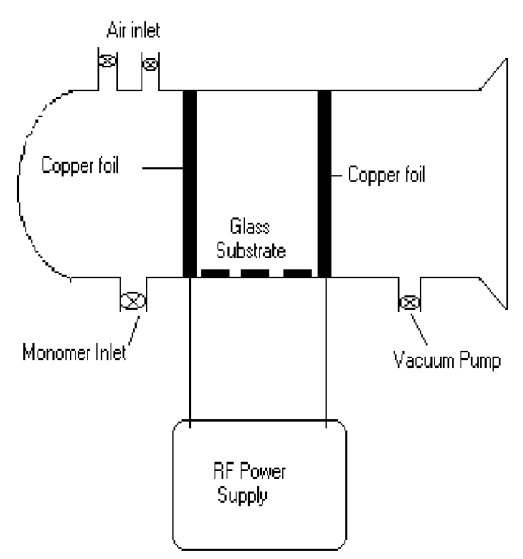

(b)

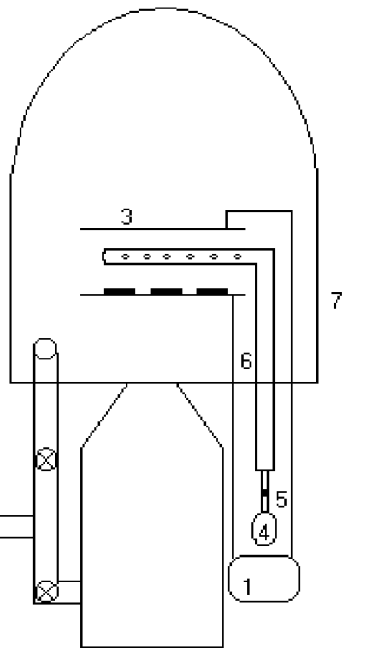

1. A.C Power Supplu

2. Rotary Pump

3. Electrodes

4. Nonomer Holder

6. Monomer Sprayer

7. Bell jar

Figure 1. (a) rf and (b) a.c. plasma polymerization set-ups. 


\section{Results and discussion}

\section{$3.1 \quad$ FTIR studies}

Figure 2 shows the FTIR spectrum of monomer aniline and polyaniline prepared under a.c. and rf conditions. Analysis of the FTIR spectra of these thin film samples indicate the presence of aromatic ring stretching $\left(1600 \mathrm{~cm}^{-1}\right)$ and $\mathrm{C}-\mathrm{C}$ stretched vibration $\left(1500 \mathrm{~cm}^{-1}\right)$ even after polymerization. It can also be seen that the aromatic ring is intact in post plasma polymerized films. This increases the stability of polymer thin films. It is evident from the spectra that the aromatic $\mathrm{CN}$ bonding around $1240 \mathrm{~cm}^{-1}$ which is principal to all polymerized samples is found to be blue shifted with respect to the monomer.

A quick comparison between the spectra of a.c. and $\mathrm{rf}$ thin films exhibit marked structural difference between the polymers evolved by both polymerization routes viz. $\mathrm{rf}$ and a.c. It is observed that $\mathrm{NH}$ stretching $\left(3220 \mathrm{~cm}^{-1}\right)$ is not very prominent in the a.c. plasma polymerized samples but its presence is seen in the rf plasma polymerized samples. A peak at $3038 \mathrm{~cm}^{-1}$ corresponding to $\mathrm{CH}$ stretching is retained in the polymerized samples. The peak at around $2960 \mathrm{~cm}^{-1}$ corresponding to $\mathrm{NH}$ asymmetric stretching in the monomer is found at $2928 \mathrm{~cm}^{-1}$ in a.c. and $2970 \mathrm{~cm}^{-1}$ in rf plasma polymerized samples, respectively. The $\mathrm{CH}$ inplane deformation $\left(1175 \mathrm{~cm}^{-1}\right)$ is found to be retained in the a.c. plasma polymerized samples but is seen shifting to $1105 \mathrm{~cm}^{-1}$ in $\mathrm{rf}$ plasma polymerized samples (Bellami 1962).

The FTIR studies and further analysis establishes the fact that structural difference in the a.c. and $\mathrm{rf}$ plasma polymerized aniline thin film samples do exist. It can also be seen that doping with iodine modifies the bond length and facilitates shifting of functional groups.

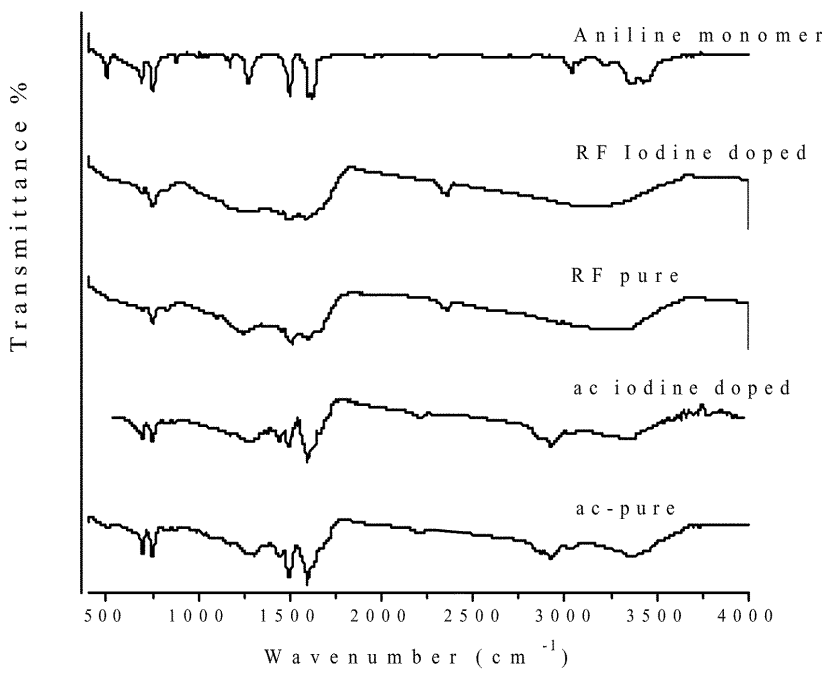

Figure 2. FTIR spectrum of plasma polymerized polyaniline thin films.

\section{$3.2 U V$-vis-NIR studies}

The photon absorption in many amorphous semiconductors is observed to obey the Tauc relation (Tauc et al 1970)

$$
\alpha h v=B\left(h \gamma-E_{\mathrm{opt}}\right)^{n},
$$

where $\alpha$ is the absorption coefficient, $h \gamma$ the photon energy, $B$ a constant and here $n=1 / 2$ for direct and $n=2$ for indirect transitions. The value of $n$ for all the samples were estimated from the slope of the $\log \alpha$ vs $\log h v$ plots by taking a linear fit. The values corresponding to $n$ are $2 \cdot 2,2 \cdot 22,2 \cdot 0$ and 2.05 for $\mathrm{rf}$ pristine, $\mathrm{rf}$ iodine doped, a.c. pristine and a.c. iodine doped samples, respectively. The value of the density of state function can be approximated to 2 and the transitions found in all the samples are indirect.

The UV-vis-NIR spectra of the polyaniline thin films are depicted in figure 3 . The absorption coefficient is plotted in the $y$-axis and energy $h v$ in the $x$-axis and the optical band gap of pure and doped polyaniline thin films are determined from these plots (figure 4).

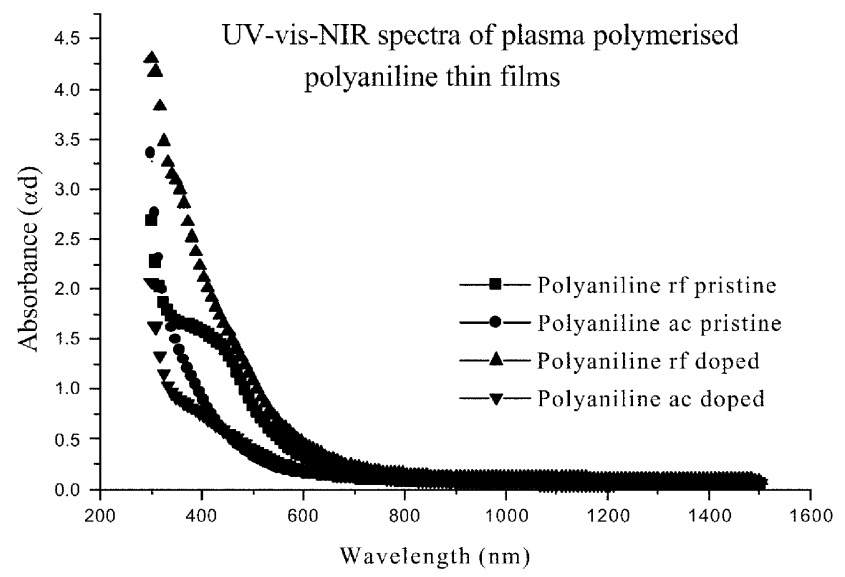

Figure 3. Absorption spectra of a.c. and rf plasma polymerized aniline thin films.

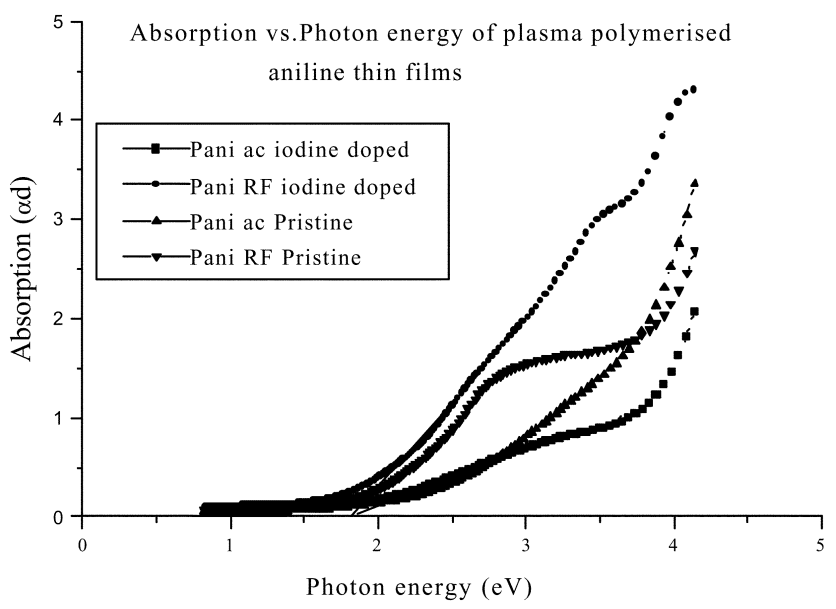

Figure 4. Absorption vs photon energy of a.c. and rf plasma polymerized aniline thin films. 
Table 1. Comparison of optical transition energies of polyaniline samples prepared by $\mathrm{rf}$ and a.c. plasma polymerization.

\begin{tabular}{lccccc}
\hline & \multicolumn{4}{c}{ Optical transition energies (eV) } \\
\cline { 2 - 3 } \cline { 5 - 6 } \cline { 5 - 6 } Polyaniline & $\begin{array}{c}\text { First } \\
\text { transition }\end{array}$ & $\begin{array}{c}\text { Pecond } \\
\text { transition }\end{array}$ & & $\begin{array}{c}\text { First } \\
\text { transition }\end{array}$ & $\begin{array}{c}\text { Second } \\
\text { transition }\end{array}$ \\
\cline { 2 - 3 } RF plasma polymerized & 2.2 & & & 1.9 & 2.6 \\
A.C. plasma polymerized & 2.3 & 3.4 & & 1.8 & 3.6 \\
\hline
\end{tabular}

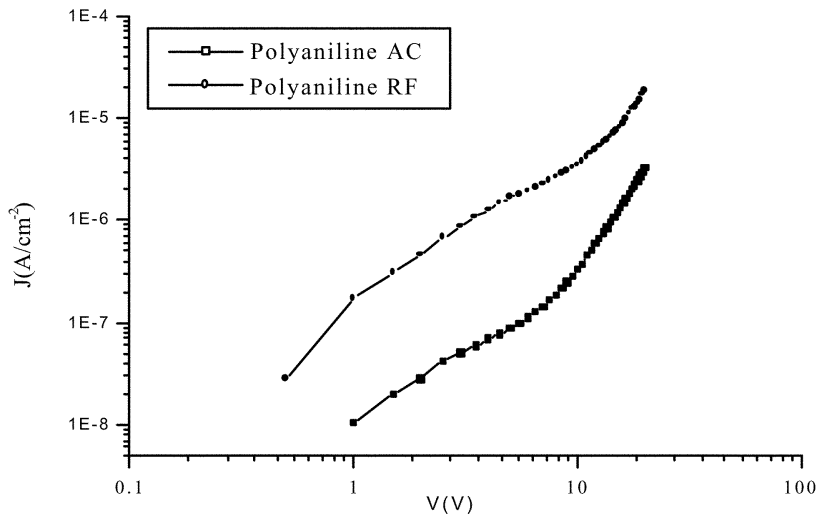

Figure 5. Comparison of conductivity of rf and a.c. plasma polymerized thin films in their pristine forms.

The optical transition energies (optical band gap) corresponding to the films prepared under various deposition conditions are given in table 1 . It can be seen that the optical transition energies of $\mathrm{rf}$ polyaniline (PANI) films are considerably different with respect to their a.c. counterpart. It is also evident from the table that the iodine doping decreases the optical band gap from $3.6 \mathrm{eV}$ to $2.8 \mathrm{eV}$ and $2.2 \mathrm{eV}$ to $1.9 \mathrm{eV}$ in the case of a.c. and rf plasma polymerized thin films, respectively. Also the photon energy of the second transition is different in the doped samples. A reduction of $0.8 \mathrm{eV}$ and $0.3 \mathrm{eV}$ in the optical transition energies are noticed with respect to the pure and doped $\mathrm{rf}$ plasma polymerized samples. However, in the case of a.c. plasma polymerized polyaniline thin films, the optical transition found at $3.4 \mathrm{eV}$ is shifted to $3.6 \mathrm{eV}$ as a result of iodine doping whereas the optical transition found at $2.3 \mathrm{eV}$ reduced to $1.8 \mathrm{eV}$. The reduction in the optical band gap is probably due to the modification of the polymer structure (Wise 1998).

The reduction of band gap in the rf polymerized samples arises out of extended conjugated structure evolved in the rf polymerization mechanism. Doping induces a structural ordering of the polymers due to the incorporation of the charged species (Huang and MacDiarmid 1997). There are signatures supporting these changes in the UVvis- and FTIR spectra.

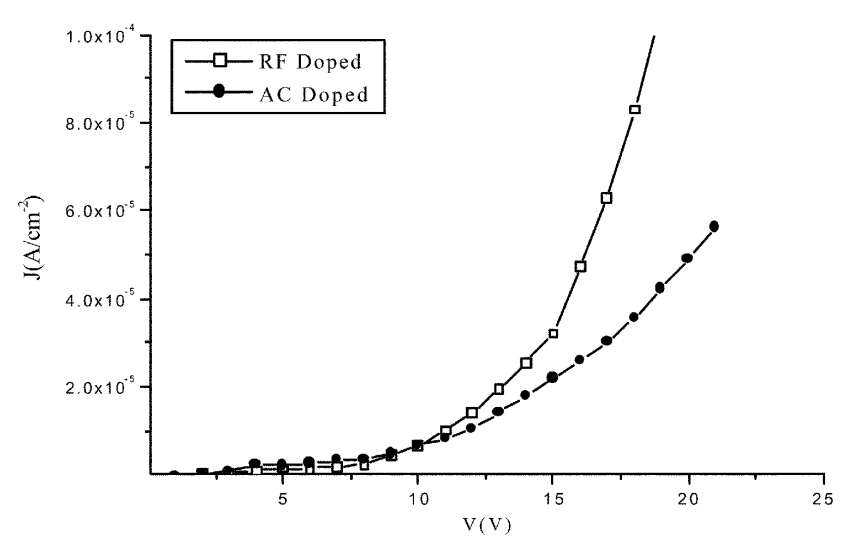

Figure 6. The current density, $J$ vs $\log V$ plot of rf and a.c. plasma polymerized thin films in the iodine doped forms.

\subsection{Conductivity measurements}

The $\log J$ vs $\log V$ plot of rf and a.c. plasma polymerized thin films are shown in figure 5. The dependence of current density with the variation of electric field is evident from figure 5. The mechanism of conduction of films prepared by rf and a.c. plasma polymerization is found to be different and conductivity of the rf plasma polymerized thin films is higher than that of the a.c. plasma polymerized films. Also the value of current density is enhanced by iodine doping. This can be attributed to the evolution of conjugated structure during the rf plasma polymerization due to the benzene ring opening in the presence of iodine (Blom et al 1997).

The variation of conductivity of the samples of thickness $(160 \pm 10 \mathrm{~nm})$ for the pristine and iodine doped forms at various voltages are depicted in figure 6 . The conductivity is enhanced by an order of magnitude in the case of both rf and a.c. plasma polymerized thin films. Also the increase of current density with respect to the increase in biasing voltage is more rapid in the case of rf films. It is to be noted that this rapid increase in current density is observed in the rf films in the pristine form also. In this case the field induced non-linear enhancement of the conductivity attains its trap filled region for lower applied voltage than that of a.c. plasma polymerized films. Typically the mechanism of conduction observed in these films 
is space charge limited conduction (SCLC). The double $\log (J-V)$ plot of the films shows space charge limited conduction which shows a linear region which can occur only at a low bias voltage where the equation of conductivity will follow the ohm's law

$$
J=\mu n_{0} e \frac{V}{d}
$$

where $e$ is the electronic charge, $V$ the voltage applied and $d$ the inter electrode distance or the film thickness. At higher voltages, SCLC current density is given by the Mott-Gurney relation

$$
J=\frac{9}{8} \varepsilon_{0} \varepsilon_{\mathrm{r}} \mu_{\mathrm{p}} \frac{V}{d^{3}},
$$

where $\varepsilon_{0} \varepsilon_{\mathrm{r}}$ is the permittivity of the polymer, $\mu_{\mathrm{p}}$ the carrier mobility and $d$ the thickness of the device. From the slope of the $\log J-\log V$ plot, it is observed that the current density, $J$, depends quadratically on the voltage, $V$, which is a characteristic of SCLC and after this region the quadratic dependence of $J$ on $V$ increases to a trap filled limit (TFL).

Polyaniline is generally considered as a $p$-type conductor. The iodine like electron acceptor dopant reduces the binding energy of the polymer (Xu et al 2002). This reduction of binding energy results in an enhanced mobility of the carriers and the conductivity is enhanced further. It is noteworthy that a rapid increase in the current density is observed in the case of pristine and rf plasma polymerized films. This observation finds complimentary evidences from band gap studies and it can be seen that optical band gap generally decreases with iodine doping.

\section{Conclusions}

In situ doping of iodine reduces the band gap considerably in both $\mathrm{rf}$ and a.c. plasma polymerized aniline thin films. The reduction in the optical band gap is more pronounced in the case of rf plasma polymerized aniline films and is attributed to the conjugation arising out of the increased power during rf plasma polymerization. The electrical conductivity of polyaniline films is also modified due to the lowering of the binding energy of the polymer resulting out of the iodine incorporation in its backbone.

\section{Acknowledgements}

(MRA) acknowledges the Department of Space, Government of India, for the financial assistance received in the form of a project under ISRO-RESPOND, Government of India (File. No. 10/03/354 dtd 23-02-1999). (USS) acknowledges CUSAT for a fellowship. Authors thank P M Ratheesh Kumar, Department of Physics, CUSAT, for the thickness measurement of thin films.

\section{References}

Bellami L J 1962 Infrared spectrum of complex molecules (New York: John Wiley and Sons)

Blom P W M, de Jong M J M and Van Munster M G 1997 Phys. Rev. $\mathbf{B 5 5} 656$

Huang F and MacDiarmid A G 1997 Appl. Phys. Lett. 712415

Joseph Mathai C, Saravanan S, Anantharaman M R, Venkatachalam S and Jayalekshmi S 2002 J. Phys. D: Appl. Phys. 35 240, 2206

Li K and Meichsner J 2001 J. Phys. D: Appl. Phys. 341318

MacDiarmid A G 2001 Curr. Appl. Phys. 269

Paterno L G, Manolache S and Denes F 2002 Synth. Met. 13085

Saravanan S, Joseph Mathai C, Venkatachalam S and Anantharaman M R 2004 New J. Phys. 664

Skotheim Terja A (ed.) 1986 Hand book of conducting polymers (New York: Marcel Dekker Inc.)

Tauc J, Menth A and Wood D 1970 Phys. Rev. Lett. 25749

Wise Donald L (ed.) 1998 Photonic polymer systems, fundamentals, methods and applications (New York: Marcel Dekker Inc.)

Xu B, Jaewu Choi, Carus A N and Dowben P A 2002 Appl. Phys. Lett. 804345

Yasuda H 1985 Plasma polymerization (New York: Academic Press Inc.) 